\title{
Diabetic lumbosacral radiculoplexus neuropathy: a postmortem studied patient and review of the literature
}

\author{
David S. Younger
}

Received: 23 December 2010/Revised: 22 January 2011/ Accepted: 26 January 2011/Published online: 17 February 2011 (C) The Author(s) 2011. This article is published with open access at Springerlink.com

\section{Dear Sirs,}

Peripheral neuropathy is an important complication of diabetes mellitus. A particular type of proximal diabetic neuropathy, lumbosacral radiculoplexus neuropathy (DLSRPN), presents with pelvi-femoral pain followed by weakness, beginning focally in the upper leg or thigh with spread to the contralateral limb, and variable weight loss [1]. Ischemic nerve injury due to microscopic vasculitis has been a widely postulated unifying hypothesis of DLSRPN and derived from one well studied postmortem case reported 4 decades ago, the finding of microscopic vasculitis in proximal or distal cutaneous nerve biopsy tissue and the favorable response to immunotherapy.

A 59 year old man with diabetes mellitus for 15 years treated with oral hypoglycemic medication noted left thigh pain, paresthesia, and impotence commencing in January 1995. This was followed by sensory changes and weakness first in the left leg then in the other in a stepwise fashion leading first to the necessity of a cane, then walker accompanied by a $15 \mathrm{lb}$ weight loss, until he was essentially bed-bound and admitted to the hospital in June 1995. Admission neurological examination showed wasting and near-flail weakness in the legs from the thighs to the toes with rare limb fasciculation, mild distal weakness of the hands in the distribution of the distal median and ulnar nerves, mild stocking sensory loss to light touch, vibratory and cold temperature stimulation to just above the knee, areflexia, and otherwise intact cranial nerves and cognition.

\section{S. Younger $(\square)$}

Department of Neurology, New York University

School of Medicine, 333 East 34th Street,

Suite 1J, New York, NY 10016, USA

e-mail: david.younger@nyumc.org
Admission laboratory studies showed erythrocyte sedimentation rate $60 \mathrm{~mm} /$ hour (normal $<20$ ), fasting blood glucose $250 \mathrm{mg} / \mathrm{dL}$ (normal <105), and hemoglobin A1C level $7.1 \%$ (normal <6). Causes of neuropathy other than diabetes mellitus were excluded by appropriate investigations. Nerve conduction studies and electromyography (EMG) of the right arm and leg showed axonal neuropathy with acute and chronic denervation in proximal and distal limb muscles including lumbosacral paraspinal muscles. Cerebrospinal fluid showed total protein $122 \mathrm{mg} / \mathrm{dL}$ (normal <45) and glucose $88 \mathrm{mg} / \mathrm{dL}$ with 12 white blood cells/ $\mathrm{mm}^{3}$ (normal $<5$ ). Right sural nerve biopsy showed features of microvasculitis (MV). Inflammatory cells surrounded a small epineurial artery with extension into the vascular wall, with reactive luminal connective tissue suggesting recanalization of a thrombus. An adjacent nerve fascicle showed marked loss of myelinated nerve fibers. The patient was treated for painful diabetic lumbosacral plexopathy and peripheral nerve vasculitis according to prevailing standards with $2 \mathrm{~g} / \mathrm{kg}$ intravenous immunoglobulin for 5 days, followed by $750 \mathrm{mg}$ of intravenous cyclophosphamide and $1,000 \mathrm{mg}$ of methylprednisolone intravenously for 3 additional days. This was followed by acute tubular necrosis, increasing lethargy, unresponsiveness, and aspiration pneumonia requiring mechanical ventilation. He expired 4 weeks after admission.

General autopsy showed no evidence of systemic or peripheral nerve vasculitis. The brain showed diffuse loss of neurons in all sampled cortical areas, including the cerebellum, consistent with anoxia secondary to cardiac arrest. Sections of extradural lumbar plexus, sciatic, and femoral nerve tissue showed perivascular epineurial inflammation with infiltration of adjacent endoneurium (Fig. 1a, b).

Perivascular inflammation, not MV, was noted in postmortem nerve tissue in our patient. Although the 


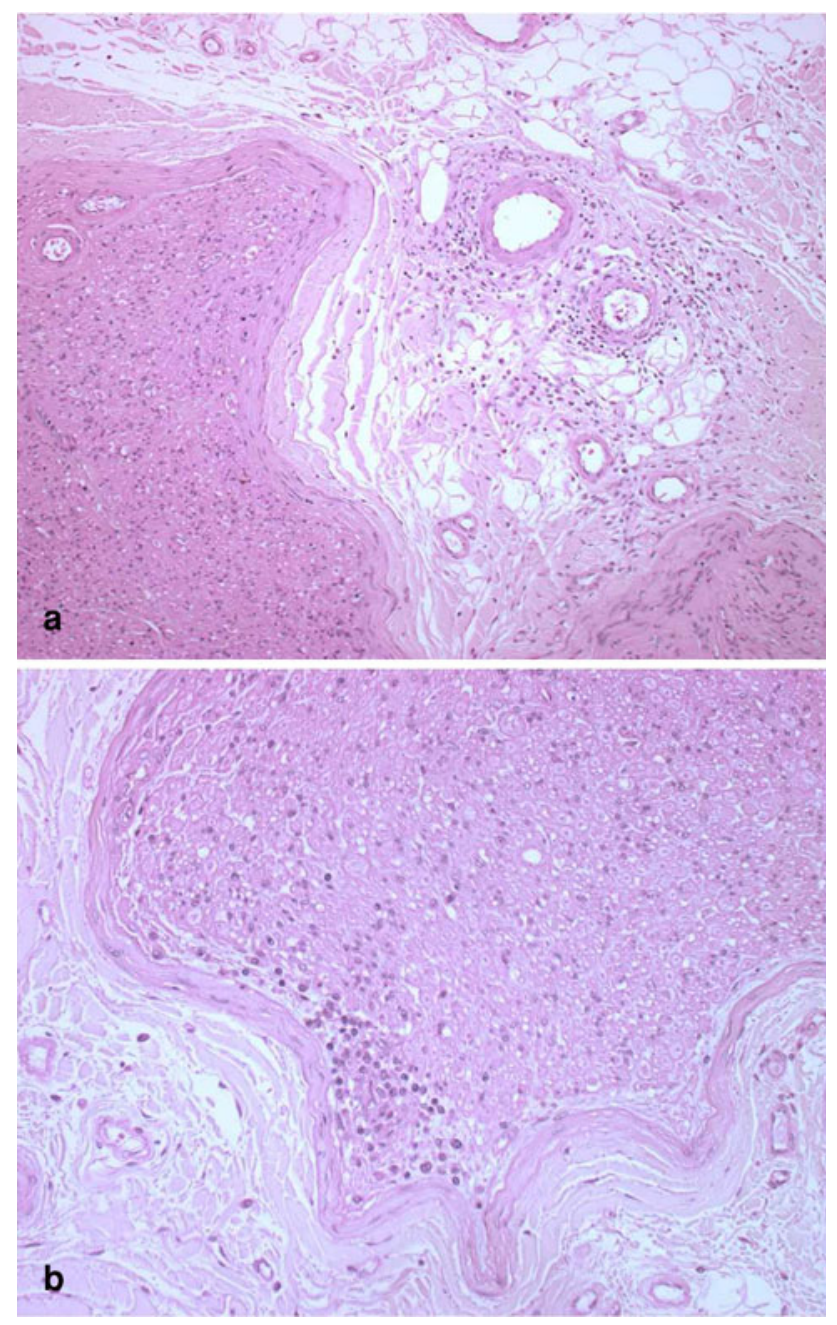

Fig. 1 Postmortem histopathology. a Transverse section of the left sciatic nerve shows perivascular chronic inflammation surrounding small blood vessels of the epineurium. b Transverse section of the left femoral nerve in addition shows perivascular chronic inflammation in the subperineurial area. Inflammatory cells infiltrate the adjacent endoneurium (Paraffin, H\&E, 200X)

significance of our findings is not well understood, they do suggest the contribution of vascular autoimmune factors in the etiopathogenesis of DLSRPN, but do not provide clear evidence of ischemic nerve injury due to microscopic vasculitis in this disorder.

The history of DLSRPN embodies more than a century of insights into proximal diabetic neuropathy (PDN), diabetic amyotrophy, and peripheral nerve vasculitis. An ischemic etiopathogenesis of DLSRPN was first suggested by Raff and colleagues [2] in a newly diagnosed noninsulin dependent diabetic with mononeuritis multiplex presenting with acute asymmetrical leg pain and weakness, bilateral distal sensory disturbances, and reduced leg reflexes. Postmortem examination showed a multitude of unilateral small ischemic infarctive lesions of the proximal major nerve trunks of the leg and lumbosacral plexus.
Further proof of the ischemic inflammatory basis of DLSRPN stemmed from analysis of proximal and distal cutaneous nerve biopsy. In 1984, Bradley and coworkers [3] delineated the syndrome of painful lumbosacral plexopathy with elevated erythrocyte sedimentation rate among six patients, three of whom were diabetic including one newly diagnosed, with perivasculitis (PV) in sural nerve biopsies. In the same period, Johnson and coworkers [4] noted focal fascicular lesions distributed in proximal lumbosacral plexus trunks of 18/32 samples obtained at autopsy from diabetic patients, a quarter of whom were insulin-dependent, without mononeuritis multiplex employing epoxy-embedded and teased nerve fiber sections. These findings suggest a possible propensity for the spontaneous evolution of DLSRPN in patients with diabetic neuropathy. A decade later, Said and coworkers [5] studied 10 non-insulin dependent diabetics with painful PDN and reported ischemic nerve lesions due to arteritis in three biopsies of the intermedius cutaneous nerve of the thigh, and four others with isolated mononuclear cell inflammation. One year later, Krendel and associates [6] identified 5 of 15 non-insulin dependent diabetics with progressive proximal asymmetric neuropathy progressing over 1-15 months punctuated by pain, weakness, and atrophy of one thigh, followed by involvement of the contralateral extremity in 2-10 weeks. Biopsy of the cutaneous branch of the femoral nerve in one patient showed epineurial PV, while sural nerve tissue in another had microfasciculation that was postulated to result from regeneration after nerve infarction. In 1996, Younger and colleagues [7] characterized the vascular endoneurial and epineurial inflammatory lesions among 12 patients with stepwise or slowly progressive proximal weakness, wasting, and pain, and axonopathy on electrodiagnostic studies indicative of DLSRPN. Six nerves showed epineurial MV and six had epineurial PV comprised of cytotoxic/suppressor $\mathrm{T}$ cells with activated endoneurial lymphocytes that expressed immunoreactive cytokines, major histocompatibility class II antigens, endoneurial and epineurial C3d and C5b-9 complement. The nerve tissue of two patients with $\mathrm{MV}$ in addition had focal pathology indicative of ischemia. In 1998, Llewelyn and colleagues [8] studied 15 patients including one with insulin-dependent diabetes and PDN manifested by subacute asymmetric pain, weakness, and wasting of the legs. Epineurial MV was noted in three of 15 femoral intermedius cutaneous nerve biopsies comprised of helper $\mathrm{T}$ cells and cytotoxic/suppressor $\mathrm{T}$ and $\mathrm{B}$ cells, with a single occluded vessels in two, and T cell MV in the sural nerve and vastus lateralis muscle of the another patient.

Dyck and colleagues [9] later reported perivascular inflammation in all 33 distal cutaneous nerves in DLSRPN, $15(45 \%)$ of which infiltrated the vessel wall, and MV in two nerves with histological features of ischemic nerve injury. One year later, Kelkar and colleagues [10] 
described 16 patients with PDN and EMG findings of acute spontaneous activity in two proximal leg muscles innervated by different nerves, including but not limited to paraspinal muscles indicative of DLSRPN; all were noninsulin dependent, with functional impairment that varied from three patients who were wheelchair bound, four necessitating a walker, and eight patients requiring support of a cane to ambulate at presentation. Fourteen patients underwent femoral cutaneous nerve biopsy and two underwent sural nerve biopsy. Nerve biopsy pathology showed epineurial MV comprised of polymorphonuclear cells in postcapillary venules of the femoral cutaneous nerve in four patients, and lymphocytic MV of the sural nerve of six patients, including one nerve with occlusion and recanalization of an epineurial vessel. There was binding of immunoreactive IgM and activated complement protein in the subperineurial space and endoneurium consistent with immune-mediated ischemic injury.

The past 3 decades have witnessed a critical appraisal of the immunotherapeutic management of DLSRPN. Bradley and coworkers [3] administered corticosteroids ranging from $60 \mathrm{mg}$ daily to $80 \mathrm{mg}$ of alternate day prednisone alone in one patient, and in association with cyclophosphamide $100 \mathrm{mg}$ daily in two others, with improvement over 6 weeks often with a decrease in the erythrocyte sedimentation rate. A decade later the same authors [11] treated the disorder with $2 \mathrm{~g} / \mathrm{kg}$ body weight of intravenous immune globulin (IVIg) over 5 days followed by single monthly treatments for 3 months, the endpoint of which was improved Medical Research Council (MRC) graded strength that enabled ambulation. In the same year, 1995, Said and colleagues [5] treated two of 10 affected patients with prednisone $1 \mathrm{mg} / \mathrm{kg}$ /day for 6 weeks prompted by the presence of lymphocytic vasculitis in epineurial blood vessels with asymmetrical nerve lesions and centrofascicular axonal loss along the intermedius nerve of the thigh in one, and another with occlusion of a perineurial blood vessel and a small mononuclear inflammatory cell infiltrate along the superficial sensory branch of the peroneal nerve. Both patients had improved pain along with corticosteroid induced hyperglycemia, including one patient with a gastric ulcer. Seven additional patients, all without vasculitis improved spontaneously. Krendel and coworkers [6] treated five affected patients with IVIg alone in one patient, in combination with $60 \mathrm{mg}$ of prednisone in 3 patients, or with $1 \mathrm{~g}$ of intravenous cyclophosphamide in another. All five patients stopped progressing after commencing therapy and gained at least 1 grade of the MRC scale.

Most recently, Dyck and colleagues [9, 12] noted equally significant objective improvement in primary outcome measures of 49 patients with DLSRPN randomized to $1 \mathrm{~g} 3$ times weekly of intravenous methylprednisolone for 12 weeks, compared to 26 patients with DLSRPN who received placebo when analyzed at 52 [12] and 104 weeks [9]. However, the methylprednisolone treated patients reported a greater degree of symptom improvement as judged by changes in a neuropathy symptom change subscore for pain. A randomized, double-blind placebo-controlled trial of IVIg on recovery time of patients with PDN and EMG evidence of proximal lower limb plexus or radicular denervation that commenced in 1999 [13] is still ongoing but no longer recruiting study participants, and has yet to publish its findings.

In summary, DLSRPN is a well recognized, painful, asymmetrical, immune mediated neuropathy of the lower limbs associated generally with non-insulin dependent diabetes, weight loss, and significant morbidity. The disorder has evolved along different nomenclature and eponymic terminology reflecting the diversity in opinions concerning the anatomic localization and underlying etiopathogenesis. The clinicopathological findings in the present patient were consistent with a mechanism of ischemic nerve injury due to microscopic vasculitis. Neurologists faced with the challenge of treating DLSRPN must choose from among available immune mediated interventions, weighing the inherent risks, benefits, and anticipated efficacy, and tailored to the clinical, electrophysiological, and cutaneous nerve biopsy findings to optimize recovery and forestall severe neurological disability.

Acknowledgment Arthur P. Hays provided Fig. 1.

Conflict of interest The author has no conflict of interest.

Open Access This article is distributed under the terms of the Creative Commons Attribution Noncommercial License which permits any noncommercial use, distribution, and reproduction in any medium, provided the original author(s) and source are credited.

\section{References}

1. Dyck PJB, Norell JE, Dyck PJ (1999) Microvasculitis and ischemia in diabetic lumbosacral radiculoplexus neuropathy. Neurology 53:2113-2121

2. Raff MC, Sangalang V, Asbury AK (1968) Ischemic mononeuropathy multiplex associated with diabetes mellitus. Arch Neurol 18:487-499

3. Bradley WB, Chad D, Verghese JP et al (1984) Painful lumbosacral plexopathy with elevated erythrocyte sedimentation rate: a treatable inflammatory syndrome. Ann Neurol 15:457-464

4. Johnson PC, Doll SC, Cromey DW (1986) Pathogenesis of diabetic neuropathy. Ann Neurol 19:450-457

5. Said G, Goulon-Goeau C, Lacroix C et al (1994) Nerve biopsy findings in different patterns of proximal diabetic neuropathy. Ann Neurol 35:559-569

6. Krendel DA, Costigan DA, Hopkins LC (1995) Successful treatment of neuropathies in patients with diabetes mellitus. Arch Neurol 52:1053-1061 
7. Younger DS, Rosoklija G, Hays AP, Trojaborg W, Latov N (1996) Diabetic peripheral neuropathy: a clinicopathologic and immunohistochemical analysis of sural nerve biopsies. Muscle Nerve 19:722-727

8. Llewelyn JG, Thomas PK, King RHM (1998) Epineurial microvasculitis in proximal diabetic neuropathy. J Neurol 245:159-165

9. Dyck PJB, O'Brien P, Bosch P et al (2006) The multi-center double-blind controlled trial of IV methylprednisolone in diabetic lumbosacral radiculoplexus neuropathy. Neurology 66(Suppl 2):A191

10. Kelkar P, Masood M, Parry GJ (2000) Distinctive pathological findings in proximal diabetic neuropathy (diabetic amyotrophy). Neurology 55:83-88
11. Varma A, Bradley WG (1994) High-dose intravenous immunoglobulin therapy in chronic progressive lumbosacral plexopathy. Neurology 44:248-250

12. Dyck PJB, O'Brien PC, Bosch EP et al (2005) Results of a controlled trial of IV methylprednisolone in diabetic lumbosacral radiculoplexus neuropathy (DLRPN): a preliminary indication of efficacy. J Periph Nerv Syst 2005 101(1):21

13. Windebank A. Study Chair. Randomized study of intravenous immunoglobulin (IVIg) in patients with subacute proximal diabetic neuropathy. FDA Office of Orphan Products Development. Mayo Clinic, clinicaltrials.gov/show/NCT 00004407 